\title{
Diagnóstico, evaluación y tratamiento no farmacológico del paciente con sobrepeso u obesidad
}

\author{
Mónica Manrique ${ }^{1}$, María Pía de la Maza², \\ Fernando Carrasco ${ }^{3}$, Manuel Moreno ${ }^{4}$, Cecilia Albala ${ }^{2}$, \\ Jaime García ${ }^{5}$, Jaime Díaz ${ }^{6}$, Claudio Liberman 7 . \\ Statement about diagnosis \\ assessment and non pharmacological \\ treatment of obesity
}

The risk of complications of obesity is proportional to body mass index and is higher in severe or morbid obesities and when abdominal or visceral fat is predominant. In Chile the prevalence of obesity is increasing. According to the World Health Organization, obese subjects must reduce at least a $5 \%$ of their weight to reduce the risk of complications. Although this amount of reduction is seldom achieved with non pharmacological treatments, better results are obtained with multidisciplinary approaches that include a medical, psychosocial and laboratory assessment, to determine obesity level and different factors involved and the associated complications. In a second stage, goals of treatment are set and a personalized treatment is designed including dietary changes and physical activity. The aim is to obtain perdurable lifestyles modifications (Rev Méd Chile 2009; 137: 963-71).

(Key words: Body mass index; Life style; Obesity)

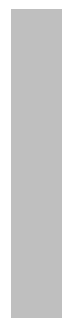

\begin{abstract}
Recibido el 8 de mayo, 2009. Aceptado el 18 de mayo, 2009.
Documento de consenso del Grupo de Estudio para la Obesidad, de la Sociedad Médica de Santiago.

${ }^{1}$ Instituto Médico Nutramed. ${ }^{2}$ Instituto de Nutrición y Tecnología de los Alimentos, Universidad de Chile, Santiago, Chile. ${ }^{3}$ Departamento de Nutrición, Facultad de Medicina, Universidad de Chile, Santiago, Chile. ${ }^{4}$ Departamento de Nutrición, Diabetes y Metabolismo, Pontificia Universidad Católica de Chile, Santiago, Chile. ${ }^{5}$ Centro Médico Capredena, Santiago. ${ }^{6}$ Unidad de Diabetes, Hospital San Juan de Dios, Santiago, Chile. ${ }^{7}$ Departamento de Endocrinología, Hospital Clínico de la Universidad de Chile, Santiago, Chile.
\end{abstract}

$\mathrm{L}^{\mathrm{a}}$ a obesidad ha adquirido proporciones epidémicas a nivel global. En la actualidad, con más de un billón de adultos con sobrepeso y obesidad y por lo menos 300 millones de obesos, es uno de

Correspondencia a: Dra. Mónica Manrique E. Instituto Médico Nutramed. Av. Kennedy 5757, of. 707 Santiago. Fono: 2452794. Fax: 2452794. E mail: mmanrique@nutramed.cl los mayores contribuyentes a la carga global de enfermedad y discapacidad. Su frecuente asociación con comorbilidades, alto costo social y económico y la complejidad del tratamiento, la convierten en un problema prioritario de salud pública ${ }^{1,2}$.

Numerosos estudios epidemiológicos muestran que, en los adultos, la obesidad se asocia a un aumento de los índices de mortalidad y 
constituye un importante factor de riesgo de hipertensión, diabetes, dislipidemias, cardiopatía coronaria, apnea del sueño y otras enfermedades crónicas como colelitiasis, reflujo gastroesofágico, esteatohepatitis no alcohólica, estasia venosa, algunos tipos de cáncer, enfermedades osteoarticulares, alteraciones psicológicas, infertilidad y complicaciones ginecoobstétricas. En los casos más severos (obesidad mórbida y mega-obesidad) se intensifican estas complicaciones, las cuales determinan un aumento significativo en el riesgo vital.

Estudios en diversos países demuestran el gran impacto económico en costos directos (consultas, medicamentos y complicaciones) e indirectos (días no trabajados y pensiones) de esta patología, lo que representa valores que fluctúan entre $2 \%$ y $7 \%$ del gasto destinado a salud, superando incluso al gasto global por tratamiento del cáncer $0 \mathrm{al}$ daño producido por tabaco ${ }^{3-5}$.

En Chile la transición epidemiológica y nutricional ha ocurrido en forma muy rápida ${ }^{6}$. Así, desde una alta prevalencia de desnutrición en los años 60, hemos pasado a una situación en que la obesidad constituye el más importante problema nutricional, en todas las edades ${ }^{7}$. En la última década, la prevalencia de obesidad se ha doblado en preescolares y escolares. La situación en embarazadas también ha cambiado en forma dramática, en quienes la obesidad ha aumentado de $12,9 \%$ a 32,7\%. En dos encuestas hechas en 1988 y 1992 en adultos en Santiago, la obesidad aumentó de $6 \%$ a $11 \%$ en hombres y de $14 \%$ a $24 \%$ en mujeres. Las últimas cifras de la Encuesta Nacional de Salud del año 2003 alcanzan a 23,2\% de la población ${ }^{8}$. Este aumento se explicaría en gran medida por el sedentarismo y por los cambios en los estilos de alimentación, caracterizados por el consumo de dietas ricas en energía y altas en grasa. Contribuyen a lo anterior, a pesar de tener una buena disponibilidad en nuestro medio, el escaso consumo de vegetales y frutas por parte de nuestra población ${ }^{9}$. El manejo efectivo del peso en obesos y grupos en alto riesgo de desarrollar obesidad incluye un amplio rango de estrategias, que incluyen la promoción, la prevención, el manejo de las comorbilidades y la mantención o la pérdida de peso. Aunque existe consenso en que la mejor manera de controlar el problema de la obesidad es a través de la promoción y la prevención, no puede ignorarse la situación de los 3,4 millones de sujetos obesos en el país.

De acuerdo a la Organización Mundial de Salud (OMS), el tratamiento de la obesidad debe enfocarse hacia el manejo de los factores de riesgo y la mantención del peso en el largo plazo, ya que aunque la normalización del peso rara vez ocurre, existe evidencia de que 5\%-10\% de reducción de peso, tiene un impacto significativo sobre las enfermedades asociadas ${ }^{3}$.

El tratamiento médico de la obesidad tiene en general un éxito limitado, por cuanto menos de $20 \%$ de los pacientes mantiene una reducción de $10 \%$ del peso inicial después del año, incluso en las condiciones de un estudio clínico donde se intentó maximizar la adherencia ${ }^{10}$. A nivel nacional, la reciente evaluación del primer programa piloto, que incluyó a 276 pacientes en un tratamiento multidisciplinario de 4 meses de duración, sin utilización de fármacos, detectó $42 \%$ de abandono en este lapso y $55 \%$ logró una reducción de al menos $5 \%$ del peso inicial entre los pacientes adherentes $^{11}$. Si bien hay abundante literatura acerca del tema, no existe un consenso acerca de las estrategias terapéuticas más apropiadas para la realidad nacional. Los factores antes mencionados, han motivado a este Grupo de Estudio a revisar los principales aspectos diagnósticos y terapéuticos del problema. Se trata de entregar una visión práctica y actualizada del tema, dirigida a médicos interesados en el área, basada en consensos internacionales, literatura médica especializada y la experiencia práctica de los miembros de este grupo.

\section{ESTUDIO DEL PACIENTE OBESO}

Parámetros a evaluar en el obeso

1. Grado de obesidad, distribución de la grasa corporal y composición corporal. Existen diferentes métodos para medir la composición corporal in vivo. La precisión y exactitud de estos métodos se correlacionan directamente con su costo e inversamente con su disponibilidad en la clínica.

La recomendación actual para la evaluación clínica es utilizar el índice de masa corporal ( IMC =peso, kg/talla, $\mathrm{m}^{2}$ ), el cual no está exento de limitaciones, pero tiene una buena correla- 
ción con el porcentaje de grasa corporal. En la Tabla 1 se muestra la clasificación de la OMS en relación a los rangos de IMC y riesgo global de salud $^{3}$. También pueden emplearse otras medidas antropométricas como los pliegues cutáneos (bicipital, tricipital, subescapular y suprailíaco), cuya aplicabilidad y exactitud en el paciente adulto obeso es discutible, por la variabilidad y dificultad técnica del método en pacientes con mayores grados de obesidad.

La bioimpedanciometría tetrapolar corresponde al estudio de la composición corporal por medio del análisis de la resistencia de los tejidos al paso de una corriente alterna, lo cual puede ser útil para cuantificar con mayor precisión el contenido de grasa corporal total, sin embargo está contraindicada en pacientes embarazadas y en aquellos que presentan marcapaso, y su utilidad es limitada en pacientes con trastornos importantes en la hidratación. La densitometría radiológica (DEXA: Dual Energy X-ray Absorptiometry) para evaluar la composición corporal representa una técnica de mayor precisión, pero de menor disponibilidad y mayor costo. La distribución de grasa corporal puede ser más importante que la cantidad de grasa total. Para esto se usa la medición del perímetro de cintura, ya sea aisladamente o en relación al perímetro de la cadera, mediciones ambas muy difundidas por su sencillez, ausencia de costo, fácil aplicabilidad y excelente correlación con la grasa intraabdominal medida por métodos más sofisticados, como son los estudios de imágenes. El riesgo cardiovascular se asocia particularmente a la distribución de la grasa corporal. En la Tabla 2 se resumen los puntos de corte del perímetro de cintura adoptados por la OMS, para predecir riesgo cardiovascular y metabólico $^{3}$, los que provienen de la concordancia con un IMC de 25 y $30 \mathrm{~kg} / \mathrm{m}^{2}$, de acuerdo al sexo ${ }^{12}$.

2. Factores etiológicos y condicionantes de la obesidad, antecedentes mórbidos y patologías a sociadas. Entre los antecedentes mórbidos, debe investigarse hipertensión arterial y otras enfermedades cardiovasculares, dislipidemia, diabetes mellitus tipo 2, colelitiasis, patología hepática, apnea del sueño, cáncer, patología osteoarticular, endocrinopatías, patología psiquiátrica, etc. También debe evaluarse el antecedente de tabaquismo. Son relevantes los antecedentes familiares de obesidad, diabetes, hipertensión arterial, enfermedad coronaria, dislipidemia, hipotiroidismo, cáncer, etc.

En cuanto a actividad física, es importante evaluar el grado de actividad o sedentarismo y

Tabla 1. Grado de obesidad y riesgo global de salud según $\mathrm{OM} \mathrm{S}^{3}$

\begin{tabular}{|lcc|}
\hline & IM C $\left(\mathbf{k g} / \mathbf{~}^{\mathbf{2}}\right)$ & Riesgo \\
\hline Pre-obeso & $25-29,9$ & Aumentado \\
Obesidad (clase I) & $30-34,9$ & Moderado \\
Obesidad (clase II) & $35-39,9$ & Severo \\
Obesidad mórbida (clase III) & $\geq 40$ & Muy severo \\
\hline
\end{tabular}

Tabla 2. Indicadores y puntos de corte para determinación de riesgo asociado a obesidad

\begin{tabular}{|lccc|}
\hline Indicadores & Riesgo bajo & $\begin{array}{c}\text { Puntos de corte } \\
\text { Riesgo aumentado }\end{array}$ & Riesgo muy elevado \\
\hline *Perímetro cintura $(\mathrm{cm})$ & & & \\
Hombres & $<94$ & $94-101$ & $\geq 102$ \\
Mujeres & $<80$ & $80-87$ & $\geq 88$ \\
\hline
\end{tabular}

*Fuente: OMS, $1997^{3}$. OMS se refiere a riesgo de complicaciones metabólicas asociadas a obesidad en población caucásica. 
la capacidad del individuo para tolerar la indicación de ejercicio como parte del plan terapéutico.

También debe interrogarse por el uso de medicamentos que pueden influir en la variación del peso corporal, tales como corticoesteroides y otros tratamientos hormonales, antidepresivos, antipsicóticos, hipoglicemiantes, diuréticos, betabloqueadores, anorexígenos, antihistamínicos, etc.

En la historia clínica es de especial relevancia obtener información sobre la historia del peso corporal, para reconocer la edad de inicio de la obesidad, las fluctuaciones del peso corporal, sea espontáneas o relacionadas con tratamientos y factores gatillantes de aumento de peso. De igual importancia resulta desarrollar una breve historia alimentaria interrogando por la presencia de desayuno, periodos de ayuno diurno, tipo de alimentos elegidos y patrones alterados de alimentación, como el comer nocturno, los atracones de comidas o el "picoteo". En este aspecto, puede ser de especial interés contar con la colaboración de un profesional nutricionista para el desarrollo de una encuesta de alimentación completa.

3. Examen físico completo, incluyendo parámetros antropométricos. Es fundamental registrar el peso y talla para el cálculo del IMC, la medición del perímetro de cintura, además de presión arterial y pulsos. En el examen segmentario debe buscarse acantosis nigricans en cuello y pliegues cutáneos, acrocordón cervical, bocio, nódulos mamarios, arritmias, signos de insuficiencia cardíaca y respiratoria, visceromegalias, hernias y estrías, entre otros. En las extremidades es importante consignar especialmente la existencia de edema, várices, crujido o limitación funcional articular y lesiones en los pies.

4. Exámenes de laboratorio. Tienen como objetivo detectar factores causales, investigar comorbilidad asociada a la obesidad, estimar composición corporal y evaluar la capacidad funcional del paciente.

a) En la evaluación inicial se recomienda solicitar hemograma-VHS, orina completa, perfil bioquímico, perfil lipídico, TSH, T4 libre, T3, insulinemia basal y electrocardiograma de reposo. b) En casos específicos puede ser recomendable realizar además una curva de tolerancia a glucosa con insulinemia (al menos basal y segunda hora), electrocardiograma de esfuerzo, calorimetría indirecta para medir gasto energético de reposo, cortisol libre urinario si se sospecha síndrome de Cushing, índice de andrógenos libres en casos de hirsutismo, ecotomografía de abdomen para descartar hígado graso, polisomnografía para descartar apnea del sueño.

c) En casos excepcionales se puede solicitar tomografía axial computada o resonancia nuclear magnética ante la sospecha de un tumor del sistema nervioso central o estudio genético para descartar eventuales genopatías asociadas a obesidad.

d) Para medir composición corporal en clínica se pueden utilizar la bioimpedanciometría tetrapolar o densitometría de cuerpo entero en equipos DEXA. Estas determinaciones son útiles en casos de duda diagnóstica y como parámetros objetivos de control del tratamiento.

5. Factores psicosociales. Es importante evaluar el nivel educacional, situación laboral, entomo familiary social, motivaciones para el tratamiento, antecedentes de trastomos emocionales y patología siquiátrica, incluyendo trastomos de la conducta alimentaria. Se sugiere incorporar evaluación psicológica o psiquiátrica en casos específicos y en todo paciente previo a una cirugía bariátrica. Cabe destacar la gran prevalencia de trastomos depresivos que están presentes en aquellos pacientes que consultan por obesidad, llegando a comprometer cerca de $60 \%$ de los casos, lo que adquiere mucha relevancia en la medida que la enfermedad depresiva representa por sí sola un riesgo cardiovascular significativo ${ }^{13}$.

6. Metas del tratamiento. Éste debe ser integral y multidisciplinario, el objetivo general es la reducción de al menos $5 \%$ a $10 \%$ de peso en un periodo de 6 meses, con lo cual se logra mejoría o control de comorbilidades. Se recomienda no favorecer disminuciones de peso superiores a $1 \mathrm{~kg}$ por semana después de las primeras semanas de tratamiento. Para cirugía bariátrica, en cambio, la meta es la reducción de al menos $50 \%$ del exceso de peso después de 1 año de la operación. 


\section{MANEJO NUTRICIONAL}

Los objetivos principales del manejo nutricional del paciente obeso son permitir una reducción del peso en base al compartimiento graso y mantener este peso en el largo plazo. Para perder peso es necesario inducir un balance energético negativo, es decir, que el gasto energético supere a la ingesta de energía. Esto se logra en base a dietas con un aporte calórico inferior a los requerimientos energéticos del individuo (hipocalóricas) asociado a otros cambios en el estilo de vida, que promuevan la mantención de un peso médicamente aceptable. Adicionalmente la terapia nutricional contribuye a corregir las alteraciones metabólicas frecuentemente asociadas a la obesidad, tales como hiperglicemia, hiperinsulinemia, dislipidemias e hiperuricemia, además de disminuir las cifras de presión arterial ${ }^{14}$.

Existen numerosas alternativas en la prescripción de planes de alimentación para tratar pacientes obesos, pero el desafío es lograr motivar al paciente e intentar conseguir la mayor adherencia posible a las indicaciones nutricionales. En este esfuerzo es importante una adecuada consejería nutricional y la participación del grupo familiar, para lo cual es necesario desarrollar un programa educativo sistemático.

La dieta hipocalórica debe estar adaptada a las necesidades calóricas de cada individuo (dependientes de su masa corporal, sexo, edad y actividad física), a la capacidad económica y a la disponibilidad de alimentos. Debe estructurarse en forma equilibrada y variada, de acuerdo a los hábitos y preferencias culinarias individuales. El primer paso es que un profesional nutricionista realice un interrogatorio de hábitos y conductas del comer, a través de una encuesta alimentaria, que incluya al menos el tipo y cantidad de comidas ingeridas, horarios de alimentación. Sabemos que los obesos frecuentemente subestiman su ingesta, pero aun así es de mucha utilidad la información entregada por una encuesta de recordatorio de $24 \mathrm{~h}$ en 3 días alternos o por una encuesta por tendencia de consumo.

Los requerimientos nutricionales se estiman a través de la Tasa Metabólica Basal (TMB), calculada a partir de ecuaciones como la de HarrisBenedict o las de la OMS-FAO, que tienden a sobreestimar el gasto energético o bien se puede medir, por calorimetría indirecta. Carrasco y cols $^{15}$, desarrollaron ecuaciones derivadas de calorimetrías efectuadas en 1.257 hombres y mujeres chilenas mayores de 18 años, con un amplio rango de IMC:

TMB mujeres: (P x 10,9) - (E x 2,85) + 716

TMB hombres: (P x 11,1) - (E x 2,5) + 864 ( $P=$ peso corporal en kg; $E$ =edad en años)

En forma práctica, también se puede estimar una TMB estándar de $20-25 \mathrm{kcal} / \mathrm{kg}$ peso ideal. El valor obtenido se puede multiplicar por los factores de actividad física diaria recomendados para la población general de acuerdo a la Tabla $3^{16}$, o multiplicar por un factor de 1,3 que es el factor más recomendado para sujetos obesos sedentarios ${ }^{17}$.

Una vez estimados los requerimientos energéticos del paciente, una estrategia consiste en prescribir una alimentación con 500 a $1.000 \mathrm{kcal} /$ día menos que el gasto energético total. No son recomendables las dietas de ayuno ni las dietas muy bajas en calorías ( $<800 \mathrm{kcal} /$ día) por cuanto no aseguran el aporte requerido de micronutrientes, inducen caídas importantes del gasto energético y pérdida de masa libre de grasa. Por otra parte, la adherencia a largo plazo es más difícil. El método más utilizado es restar 600 a $700 \mathrm{kcal} /$ día del gasto energético total.

Como recomendación los nutrientes pueden entregarse en base a la siguiente pauta:

a) Carbohidratos: $100 \mathrm{~g}$ como mínimo, para preservar las proteínas y prevenir la cetosis. Se recomienda evitar los carbohidratos refinados y preferir los complejos.

Tabla 3. Factores según nivel de actividad física según FAO /W H O / U N U 16

\begin{tabular}{|lccc|}
\hline & Ligera & Nivel de actividad física & M oderada \\
\hline Factor de actividad & $1,4-1,69$ & $1,7-1,99$ & Intensa \\
\hline
\end{tabular}


b) Proteínas: 0,8 a 1,5 g/ $/ \mathrm{kg} /$ día (peso ideal), especialmente aquellas de alto valor biológico. Las proteínas son necesarias para la mantención de la masa magra y además generan un mayor efecto de saciedad que otros macronutrientes.

c) Grasas: menos de 30\% del valor calórico total con distribución de ácidos grasos saturados, poliinsaturados y monoinsaturados de $7 \%, 10 \%$ y $13 \%$, respectivamente.

d) Alcohol: se recomienda no consumirlo debido a su aporte calórico $(7 \mathrm{kcal} / \mathrm{g})$, su efecto estimulante del apetito y su efecto inhibidor de la oxidación de la grasa dietaria. Por otra parte un porcentaje importante de los pacientes obesos tiene esteatohepatitis de diverso grado, que puede deteriorarse con el consumo alcohólico.

e) Vitaminas, minerales y electrolitos: sólo se considera necesario suplementarlos en dietas con menos de $1.200 \mathrm{kcal} /$ día, con el objetivo de cubrir las recomendaciones internacionales. En cuanto al sodio, se recomienda moderar el consumo, especialmente en pacientes hipertensos.

f) Fibra: 20-30 g/día, según tolerancia o $25 \mathrm{~g} /$ $1.000 \mathrm{kcal} /$ día. Se recomienda que $2 / 3 \mathrm{se}$ aporten como fibra insoluble y $1 / 3$ como soluble. El aporte de fibra permite aumentar la saciedad, disminuir la constipación y retardar la absorción de los nutrientes.

g) Líquidos: es recomendable el consumo de al menos 1,5 litros de agua al día, además del líquido que aportan los alimentos.

h) Lácteos bajos en grasa: se recomienda consumir 3 a 4 porciones medianas al día, lo que dice relación con el beneficio que ha demostrado el calcio, al disminuir el fenómeno de resistencia a la insulina ${ }^{18}$ y promover lipólisis.

En los últimos años se han popularizado las dietas desbalanceadas, es decir aquellas que minimizan la ingesta de algún macronutriente y priorizan la ingesta de los demás. Ejemplo de esto lo constituyen las dietas bajas en carbohidratos (una de las cuales fue la de Atkins en los años 60, que contenía menos de $90 \mathrm{~g} /$ día de hidratos de carbono con un aporte alto en grasas y proteínas de origen animal). Un análisis cuidadoso que comparó diferentes tipos de dietas, desde la hipograsa tradicional a la muy baja en hidratos de carbono asegurando una ingesta isoenergética, no demostró diferencias significativas en términos de reducción del peso entre ellas ${ }^{19}$, si bien la restricción de hidratos de carbono presenta ventajas en cuanto a control glicémico, niveles de insulina, triglicéridos y colesterol HDL ${ }^{20}$. Lo anterior demuestra que la prescripción "ideal" no existe, sino que ella debe adecuarse al paciente; en la medida que éste adhiera al plan de alimentación en forma mantenida se logrará inducir el balance energético necesario para reducir el peso corporal y se favorecerá un cambio de hábito alimentario para la mantención del peso a largo plazo.

Cualquiera sea la prescripción alimentaria, es útil desde el punto de vista nutricional tener en consideración algunas indicaciones prácticas para los pacientes:

1. Eliminar de la ingesta habitual alimentos con alta densidad calórica.

2. Reducir proporcionalmente los carbohidratos refinados y las grasas, especialmente las de origen animal.

3. Preferir técnicas culinarias que eviten el uso de aceite y aliñar ensaladas con cantidades moderadas de aceite vegetal, debido a que todos los tipos de aceite tienen valores calóricos similares.

4. Preferir el consumo de alimentos ricos en fibra, tales como las verduras verdes. El consumo de frutas dulces debe ser cauteloso ya que un exceso podría implicar un aumento del aporte calórico total, al igual que algunos vegetales ricos en grasas tales como palta, aceitunas y semillas (almendra, maní, nueces, etc.).

5. Mantener horarios ordenados y fijos de comida, fraccionándolas cada $4 \mathrm{~h}$, con al menos 3 comidas principales y una o 2 colaciones. Las colaciones recomendables debieran ser principalmente proteicas y no exceder las $100 \mathrm{kcal}$ por porción. Se recomienda además una cena liviana y evitar el consumo de hidratos de carbono en la noche, para favorecer la lipólisis nocturna.

6. Se puede flexibilizar la pauta de alimentación, autorizando al paciente el consumo de una comida "no permitida" 1 a 2 veces por semana, en forma controlada.

7. Otros consejos útiles para lograr una mejor adherencia al plan de alimentación son: establecer un lugar adecuado para las comidas, 
donde no existan distracciones como televisión, computadores, juegos electrónicos, evitando comer en cama. Recomendar comer lenta y pausadamente $(20$ a 30 min por comida). Utilizar como aliños hierbas y sustancias bajas en calorías que mejoren la palatabilidad de los alimentos. Utilizar un tamaño apropiado de vajilla, que permita reducir el tamaño de las porciones. Al salir a comer fuera de su casa, planificar y anticipar el tipo de alimento a seleccionar, de acuerdo a su contenido calórico. Debe tenerse en cuenta el alto aporte calórico de la mayoría de los cereales (para desayuno o colación) en nuestro medio, por lo que al promover su incorporación a la dieta, deben aconsejarse aquellos productos que cuenten con el menor porcentaje de azúcar, sal y grasa agregada ${ }^{21}$ y mayor contenido de fibra.

8. Es recomendable llevar un registro o automonitoreo de los alimentos ingeridos diariamente, actividad física realizada, estado de ánimo y agregar medición del peso corporal al menos una vez por semana. Se ha observado que un buen cumplimiento del registro alimentario se asocia a una mayor probabilidad de éxito en el largo plazo.

Considerando que no existe una diferenciación muy clara entre alimentos llamados light 0 diet, es recomendable enseñar al paciente a leer adecuadamente el etiquetado nutricional de los alimentos. La necesidad de actualizar permanentemente nuestro rotulado alimentario requiere de un diálogo fluido entre diferentes sectores, generando confianza y desarrollo, que permitan dar los pasos en este complejo tema. También es muy necesario emprender actividades de educación y promoción directa a los consumidores en los mismos lugares de expendio como supermercados, ferias o negocios de barrio, donde según estudios realizados los clientes se encuentran muy interesados en recibir esta valiosa información $^{22}$.

Idealmente las indicaciones de alimentación debieran ser desarrolladas como minutas personalizadas y explicadas al paciente con la ayuda de un profesional nutricionista y debe ser flexible en el tiempo en que el paciente está en tratamiento.
ROL DE LA ACTIVIDAD FÍSICA EN EL TRATAMIENTO DE LA OBESIDAD

El sedentarismo tiene un papel preponderante en la génesis de la epidemia de obesidad, asociado a la automatización, uso de vehículos, extensión de la jornada laboral, uso de computador, videojuegos y televisor. Más importante aún, un nivel de actividad física deficitario implica un aumento del riesgo cardiovascular aun en sujetos delgados, en cambio un adecuado estado físico constituye un factor protector, independiente del IMC, aun en pacientes obesos ${ }^{23}$.

Basado en estas consideraciones, se piensa que la promoción de la actividad física constituye un elemento fundamental tanto en la prevención de la obesidad y sus enfermedades asociadas, como en su tratamiento. Cabe destacar que el ejercicio físico contribuye a mejorar parámetros metabólicos tales como lípidos plasmáticos, tolerancia a la glucosa, niveles de insulina y cifras tensionales, entre otros beneficios. Esto es posible de lograr tanto a través de entrenamiento aeróbico como de resistencia ${ }^{24}$. Además la actividad física tiene efectos beneficiosos a nivel psicológico y social. Los objetivos del ejercicio son aminorar la pérdida de masa libre de grasa, amortiguando así la caída del gasto energético de reposo asociada a la alimentación hipocalórica y reducción de peso. Lo anterior contribuye a lograr el balance energético negativo inducido por el plan de alimentación indicado. Se ha demostrado que constituye una herramienta importante en la mantención del peso perdido, en el largo plazo, para lo cual la actividad física debe mantenerse en forma permanente.

Primero debe evaluarse la capacidad física del individuo y detectar eventuales limitaciones para efectuar actividad física, como enfermedades cardiovasculares y del aparato locomotor, que pudieran provocar efectos negativos de la terapia. Inicialmente, se prescribe una actividad física progresiva y gradual, supervisado por profesional apropiado, con el objetivo de mejorar la adaptación cardiorrespiratoria. Se recomienda evaluar a los pacientes con test de esfuerzo, con protocolo de Naughton, que informará acerca de presencia de cardiopatía isquémica, evolución de la respuesta cronótropa y presora al ejercicio, presencia de arritmias y la capacidad física. Una vez lograda 
esta primera fase, se debiera aumentar el tiempo de actividad física hasta aproximarse a $200 \mathrm{~min}$ semanales. No existe consenso acerca del tipo de ejercicio a utilizar, siendo recomendable efectuar tanto actividades aeróbicas como de resistencia muscular. En el caso del ejercicio aeróbico la intensidad requerida es de moderada a intensa ( $50 \%$ a $80 \%$ de la frecuencia cardíaca máxima, que se calcula como 220 - edad) y para el ejercicio de resistencia, se debe llegar a 50\%-60\% de la frecuencia cardiaca máxima.

Algunos consejos útiles para lograr una mejor adherencia al plan de ejercicio:

- Adaptar el tipo de ejercicio a las condiciones médicas y psicosociales del paciente.

- En pacientes con patología osteoarticular, son recomendables los ejercicios de bajo impacto 0 en el agua.

- Intentar aumentar la actividad física derivada de la vida cotidiana (subir escaleras, evitando ascensores y escalas mecánicas), disminuir horas de televisión, videojuegos y computación, y hacer actividades que correspondan a los intereses de cada individuo.

\section{APOYO PSICOLÓGICO}

El tratamiento integral de la obesidad requiere implementar medidas que conduzcan a un cambio en el estilo de vida, es decir, que faciliten en el largo plazo la mantención de condiciones que

\section{REFERENCIAS}

1. NIH Consensus Development Conference Statement. Health implications of obesity. Ann Intern Med 1985; 1973-7.

2. Chopra M, Gaibraith S, Darnton-Hill I. A global response to a global problem: the epidemic of overnutrition. Bull World Health Organ 2002; 80: 952-8.

3. WHO. Obesity. Preventing and management the global epidemic. Report of a WHO Consultation on Obesity. Geneva: WHO, 1997.

4. Aluender S, RAYNER M. The burden of overweight and obesity-related ill health in the UK. Obes Rev 2007; 8: 467-73.

5. Suluvan PW, Ghushchyan V, Wyatt HR, Hill JO. The medical cost of cardiometabolic risk factor clusters in the United States. Obesity (Silver Spring) 2007; 15: 3150-8. favorecen un peso saludable. Para esto es importante incluir en el tratamiento intervenciones psicológicas orientadas a ampliar y profundizar la imagen de la obesidad y de la vida saludable.

Esta intervención puede ser de tipo individual o grupal y puede ser entregada por psicólogos 0 profesionales de la salud entrenados en estas técnicas. Las técnicas recomendadas son de tipo cognitivo-conductual, con énfasis en modulación emocional, e intervenciones centradas en soluciones. La temática debiera incluir aspectos como: motivación por el tratamiento, conexión entre estado emocional y tipo de alimentación, manejo y expresión de emociones como estrés y ansiedad, técnicas de autorregulación y prevención de recaídas entre otras.

Dada la alta prevalencia de psicopatologías asociadas a la obesidad tales como depresión y trastornos de la conducta alimentaria, resulta de gran importancia un proceso de evaluación 0 diagnóstico diferencial previo a la intervención, y la posterior derivación a un especialista en salud mental en los casos que así lo requieran.

Estudios realizados en el último tiempo demuestran un modelo de propagación de la obesidad muy asociado al tipo de vínculo social, donde la posibilidad de desarrollar esta patología en la medida de que un amigo o pariente cercano la tenga, aumentan en forma muy significativa. Cualquier estrategia de tratamiento o de intervención en salud pública que pretenda ser efectiva debiera considerar estas nuevas observaciones ${ }^{25}$.

6. Albala C, Vio F, Kain, J Uauy. Nutrition transition in Chile. Determinants and consequences. Public Health Nutr 2002; 51: 123-8.

7. Vio F, Albala C, Kain J. Nutrition transition in Chile revisited: mid-term evaluation of obesity goals for the period 2000-2010. Public Health Nutr 2008; 11: 405-12.

8. Ministerio de Salud. Encuesta Nacional de Salud, 2003. Minsal, Chile. http:epi.minsal.cl/epi/html/invest/ENS/InformeFinalENS.pdf (Consultado el 12 de septiembre de 2008).

9. Olivares $\mathrm{S}$, Bustos N. Consumo de verduras y frutas en grupos específicos de consumidores chilenos: elementos a considerar en su promoción. Rev Chil Nutr 2006; 33: 260-4.

10. Wing RR, Phelan S. Long-term weight loss maintenance. Am J Clin Nutr 2005; 82 (Suppl 1): 222-5. 
11. Carrasco F, Moreno M, Irribarra V, Rodríguez L, Martin MA, Alarcón A et al. Evaluación de un programa piloto de intervención en adultos con sobrepeso u obesidad en riesgo de diabetes. Rev Méd Chile 2008; 136: 13-21.

12. Han TS, Van Leer EM, Seidell JC, Lean MEJ. Waist circumference action levels in the identification of cardiovascular risk factors: prevalence study in a random sample. BMJ 1995; 311: 1401-5.

13. TAPIA A, Masson L. Detección de síntomas depresivos en pacientes con sobrepeso y obesidad. Rev Chil Nutr 2006; 33: 162-9.

14. MAKRIS AP, FOSTER GD. Dietary approaches to obesity and the metabolic syndrome in overweight and the metabolic síndrome. Bray GA, Ryan DH eds., Springer 2006; 187-210.

15. Carrasco F, Reyes E, Núñez C, Riedemann $K$, Rimler O, SÁNCHEZ G. Resting energy expenditure in obese and non-obese Chilean subjects: comparison with predictive equations for the Chilean population. Rev Méd Chile 2002; 130: 51-60.

16. FAO/WHO/UNU. 2001. Human Energy Requirements: Report of a Joint FAO/WHO/UNU Expert Consultation. FAO Food and Nutrition Technical Report Series 1. Rome.

17. BRAY GA. Contemporary diagnosis and management of obesity. Handbooks in health care Co. Newtown, Pennsylvania, USA. 1998.
18. Zemel MB, Thompson W, Milstead A, Morris $K$, CAMPBELL P. Calcium and dairy acceleration of weight and fat loss during energy restriction in obese adults. Obes Res 2004; 12: 582-90.

19. Segal-Isaacson CJ, Johnson S, Tomuta V, Cowell B, Stein DT. A Randomized Trial Comparing Low-Fat and Low-Carbohydrate Diets Matched for Energy and Protein. Obes Res 2004; 12 (Suppl 2): 130S-40S.

20. KuEIN S. Clinical Trial Experience with Fat-Restricted vs. Carbohydrate-Restricted Weight-Loss Diets. Obes Res 2004; 12 (Suppl 2): 141S-4S.

21. Organización de Consumidores y Usuarios de Chile. Estudio de cereales para el desayuno. Santiago: ODECU; 2007.

22. Domper A, Zacarias I, Olivares S. Entrega de información nutricional y caracterización de la compra de alimentos en supermercados. Rev Chil Nutr 2005; 32: 142-9.

23. Leitzmann MF, Park Y, Blair A, BaLard-Barbash R, Mouw T, HoLENBECK AR. Physical activity recommendations and decreased risk of mortality. Arch Intern Med 2007; 167: 2453-60.

24. Ahmadizad S, Haghighi AH, Hamedinia MR. Effects of resistance versus endurance training on serum adiponectin and insulin resistance index. Eur J Endocrinol 2007; 157: 625-31.

25. Christakis NA, Fowler JH. The spread of obesity in a large social network over 32 years. N Engl J Med 2007; 357: 370-9. 